\title{
Scalable Community Detection over Geo-Social Network
}

\author{
Xiuwen Zheng \\ San Diego Supercomputer Center \\ University of California San Diego \\ La Jolla, CA \\ xiz675@eng.ucsd.edu
}

\author{
Qiyu Liu \\ The Hong Kong University of Science \\ and Technology \\ Hong Kong SAR \\ qliuau@cse.ust.hk
}

\author{
Amarnath Gupta \\ San Diego Supercomputer Center \\ University of California San Diego \\ La Jolla, CA \\ a1gupta@ucsd.edu
}

\begin{abstract}
We consider a community finding problem called Co-located Community Detection (CCD) over geo-social networks, which retrieves communities that satisfy both high structural tightness and spatial closeness constraints. To provide a solution that benefits from existing studies on community detection, we decouple the spatial constraint from graph structural constraint and propose a uniform CCD framework which gives users the freedom to choose customized measurements for social cohesiveness (e.g., $k$-core or $k$-truss). For the spatial closeness constraint, we apply the bounded radius spatial constraint and develop an exact algorithm together with effective pruning rules. To further improve the efficiency and make our framework scale to a very large scale of data, we propose a near-linear time approximation algorithm with a constant approximation ratio $(\sqrt{2})$. We conduct extensive experiments on both synthetic and real-world datasets to demonstrate the efficiency and effectiveness of our algorithms.
\end{abstract}

\section{CCS CONCEPTS}

- Information systems $\rightarrow$ Geographic information systems; - Theory of computation $\rightarrow$ Social networks.

\section{KEYWORDS}

Geo-Social Network, Co-located Community Detection, Computational Geometry, Big Spatial Data

\section{INTRODUCTION}

Finding densely connected structures in social networks, a.k.a., communities, has been extensively studied in past decades. Most of the prior research focus on finding communities in social networks $[1,12,13,15]$. However, some researchers [11], have argued that for location-aware applications like location-based event recommendation and market advertisement, each community of people should be not only socially connected but also be in close locational proximity to each other. Detecting such communities is called the Co-located Community Detection (CCD) problem. One reason for this increased emphasis on CCD problems is data availability - the growing usage of mobile based services offered by social media

Permission to make digital or hard copies of all or part of this work for personal or classroom use is granted without fee provided that copies are not made or distributed for profit or commercial advantage and that copies bear this notice and the full citation on the first page. Copyrights for components of this work owned by others than ACM must be honored. Abstracting with credit is permitted. To copy otherwise, or republish, to post on servers or to redistribute to lists, requires prior specific permission and/or a fee. Request permissions from permissions@acm.org.

LENS'19, November 5, 2019, Chicago, IL, USA

(C) 2019 Association for Computing Machinery.

ACM ISBN 978-1-4503-6958-9/19/11 . \$15.00

https://doi.org/10.1145/3356473.3365189 applications that allow users to publish their real-time locations. In our own prior work $[8,20]$, we investigated the formation of HIV related communities and determined that geographic proximity is a stronger predictor of community formation among users who tweet about HIV-related health issues compared to pure network proximity on Twitter.

Some researchers have considered spatial location attributes to discover co-located communities $[3,4,11,19]$. We concluded these previous work and our work as Table 1 shows. These works can be classified into three categories based on their goals: 1)Community Detection (CD): to find all co-located communities; 2) Community Search (CS) : finding personalized communities for query vertices; 3) Maximum Community Members (MCM): find the maximum colocated community with the largest number of members, which is neither CS or CD. In this paper, we solve a community detection problem to find out all co-located communities.

Table 1: Summary of existing studies.

\begin{tabular}{cccc}
\hline Spatial Constraint Def. & Algo. & CD or CS & Distance Bound \\
\hline Modify Community Criteria & Modified CNM [4] & CD & No guarantee \\
\hline Minimum Covering Circle & AppAcc [11] & CS & No guarantee \\
\hline \multirow{2}{*}{ Bounded Circle } & RotC ${ }^{+}[19]$ & CS & 1 \\
& exact & CD & 1 \\
& approx & CD & $\sqrt{2}$ \\
\hline \multirow{2}{*}{ All-pair Bound } & AdvMax [23] & CD & 1 \\
& EffiExact[3] & MCM & 1 \\
& Apx2[3] & MCM & $\sqrt{2}+\epsilon$ \\
\hline
\end{tabular}

To give the definition of co-located community, we consider social and spatial cohesiveness constraints separately. There is already a significant body of research exists on community detection and community search on pure social network [2, 6, 7, 14, 17, 18, 21], we focus primarily on the spatial constraint aspect of the CCD problem. The existing work can be categorized into four types based on their approaches to defining spatial constraint. The first technique is to define new criteria of community by integrating both social and spatial information. For example, [4] modifies the modularity function [17] by introducing a distance decay function and then provides a community detection algorithm based on fast modularity maximisation algorithm. There are two main limitations of this technique. The first and the most serious one is that it can not provide a geographic distance bound guarantee for members in a community. Secondly, it couples social and spatial information which is less flexible if users prefer other community detection techniques, e.g. $k$-core or $k$-truss decomposition.

Different from the first technique, other existing works define spatial constraint without any regard to social cohesiveness. Fang et al [11] apply the spatial minimum covering circle $[9,10]$ to ensure that each community discovered maintains high spatial compactness. It provides a community search algorithm that returns a 
$k$-core so that a spatial circle with the smallest radius can cover all community members. However, it can not guarantee a consistent distance bound for different query vertices and the case study in experiments section well presents an example to demonstrate it. Some research [3,23] apply the all-pair distance constraint which requires that the distance between any two users in a community is within a user-specified threshold. They can guarantee a bounded distance constraint for all members, however, [22] proves that the problem to enumerating all maximal clusters satisfying this spatial constraint is NP-hard. [3, 23] provide a clique-based algorithm to enumerate maximal clusters and then find $k$-cores in each cluster. Since they solve the MCM problem where only the maximum community is returned, they develop pruning rules when enumerating clusters. However, it is impractical to introduce it to solve our CD problem where all communities should be detected. Even though [3] provides an approximation algorithm based on grids, the approximation ratio is not a constant.

[19] defines spatial constraint in a similar way to our work. It applies radius-bounded circle approach which requires that any returned community can be covered geographically by a circle with user-specified radius, and it uses $k$-core to ensure social cohesiveness. It solves a community search problem which returns co-located communities for a specified query user. Different from the problem in [3][23] which is NP-hard, the problem in [19] can be solved in polynomial time. However, the approach in [19] cannot be directly modified to solve our CD problem because of time complexity issue. Given an query user, it enumerates all candidate circles with user-specified radius passing any two nearby users and finds the $k$-core containing query user in each circle. For any query user, there can be $O\left(n^{2}\right)$ candidate circles where $n$ is number of users, and thus calculating all nodes enclosed by each circle and then finding cores from them can be very time-consuming. [19] solves a community search problem, thus $n$ is practically a small value and time complexity is not a severe issue. However, in our problem, more efficient methods should be proposed.

Contributions. Our major contributions are listed as follows.

- We design a uniform framework which decouples social and spatial constraints so that users have high freedom to define social cohesiveness (e.g., $k$-truss or $k$-core) and to choose existing community detection algorithms.

- We design an efficient spatial constraint checker which return all maximal communities satisfying spatial constraint.

- To further reduce the time complexity, we design a nearlinear approximation algorithm with a constant performance guarantee $(\sqrt{2}$-bounded) for the spatial constraint checker.

- Our framework can be modified slightly to solve community search problem for any given query user.

\section{PRELIMINARIES}

In this section, we formally define our data model and problem, and present the framework for co-located community detection.

\subsection{Problem Definition}

Definition 1 (Geo-Social Network (GeoSN)). A geo-social network $(G e o S N)$ is a directed graph $G=(V, E)$ where each $v \in V$

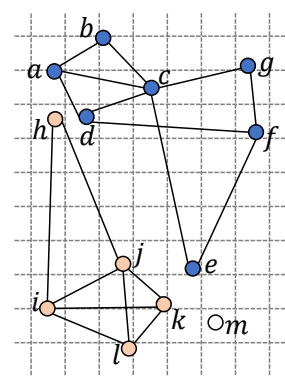

(a)

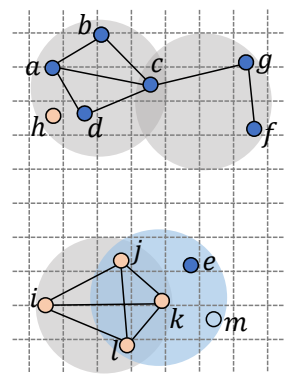

(b)
Figure 1: Illustration of geo-social network and MCCs.

denotes a user associated with a spatial location $\left(x_{v}, y_{v}\right) \in \mathbb{R}^{2}$, and E maintains the relationship (e.g., friendship) among users.

Given a geo-social network, the objective of this paper is to find all communities that simultaneously satisfy the spatial cohesiveness constraint and the social connectivity constraint. We first introduce the definition of a maximal co-located community.

Definition 2 (Maximal Co-located Community (MCC)). Given a GeoSNG, a maximal co-located community is a set of users which form a subgraph $J \subseteq G$ satisfying three constraints,

- Social Connectivity: J should satisfy a user-specified social constraint over a graph property like $k$-truss, $k$-core, etc.

- Spatial Cohesiveness: Given a distance threshold d, all the vertices of $J$ can be geographically enclosed by a circle with diameterd.

- Maximality: There does not exist a subgraph $J^{\prime} \supsetneq J$ which satisfies social connectivity and spatial cohesiveness constraints.

Definition 3 ( $d$-MCCs Detection). Given a geo-social network $G$, a distance threshold $d$ and social constraint, the problem is to find all maximal co-located communities.

EXAmPle 1. Fig. 1 (a) presents a Geo-social network where users are denoted as circles and relationships are denoted as lines. Each user is associated with a location in $\mathbb{R}^{2}$ space. Suppose that high social cohesiveness is defined as a minimum degree of at least 2, then there are two communities found in the GeoSN denoted as blue circles and orange circles respectively. Suppose that the distance threshold is set as 4 grids, then users can be divided into four overlapped groups based on their locations denoted as four shadow circles in Fig. 1 (b). Combining spatial and social information, there are two MCCs detected: $\{a, b, c, d\}$ and $\{i, j, k, l\}$.

\subsection{Framework}

To de-couple the spatial constraint from MCCs detection, we define Global Spatial Cluster merely based on spatial constraints.

Definition 4 (Global Spatial Cluster). A Global Spatial Cluster $(G S C) C \in V$ is a subset of users satisfying two constraints,

- Co-located: The cluster members of C satisfy the spatial constraint (i.e., being enclosed by a circle with diameter d).

- Maximality: IfC is an GSC, there does not exist a set of users $C^{\prime} \supsetneq C$ satisfying the co-located constraint.

Based on this definition, MCCs can be detected in three steps (Algorithm 1): (1) find all Global Spatial Clusters (line 1), (2) for 
each GSC, get the GeoSN induced by this set of users and find all local MCCs in this subgraph based on social constraint (lines 2 - 4), (3) find all MCCs by removing local MCCs which are subsets of any other MCC (FindGlobalMCC function). Note that in the first step (line 2), i.e, finding GSCs, the parameters of social constraint are also passed to the spatial algorithm so that some simple pruning techniques can be implemented.

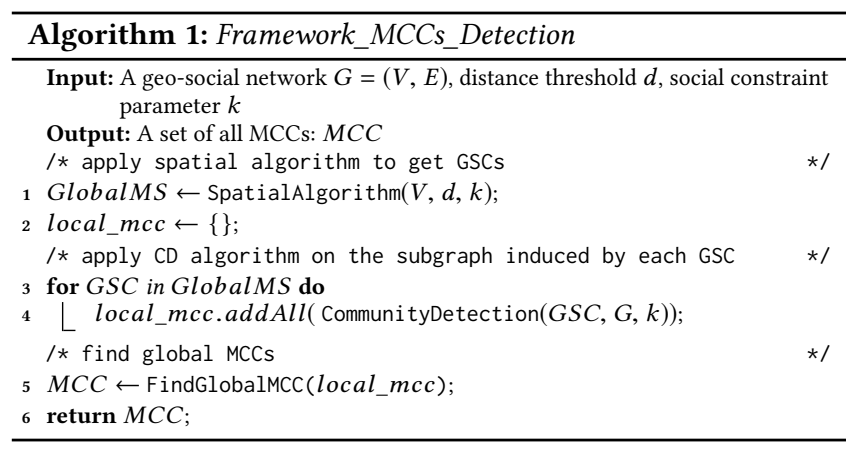

EXAMPLE 2. We still take the GeoSN in Fig. 1 (a) as an example and keep the same constraint definitions to illustrate the procedures. The first step returns four GSCs detected as the shadow circles in (b) show. In the social subgraph induced by vertices in each GSC, detect the local MCCs based on social constraint, then we get three sets: $\{a, b, c, d\},\{i, j, k, l\}$, and $\{j, k, l\}$. By calling the function FindGlobalMCC, $\{i, k, l\}$ covered in the blue shadow circle is removed from MCCs. Thus, we detect two MCCs: $\{a, b, c, d\}$ and $\{i, j, k, l\}$.

By adding two more procedures to this framework, we can easily solve the corresponding community search problem for given query user. Lines 1 and 2 find all candidate nodes that are within euclidean distance $d$ from query node $q$ and extract the small subgraph formed by this set of nodes. After applying Algorithm 1 to get all MCCs in the subgraph, line 4 filters out the MCCs that does not contain $q$.

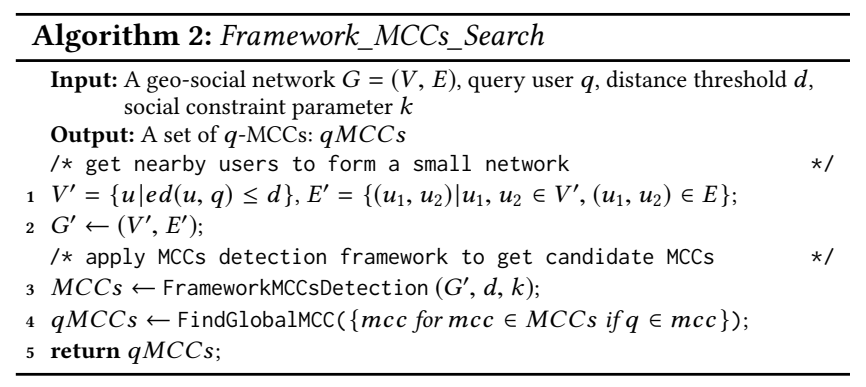

The following three sections focus on developing spatial algorithms to detect GSCs, and in the experiment section, we apply Algorithm 1 with $k$-core or $k$-truss as social constraint to detect all MCCs on five datasets.

\section{EXACT SPATIAL ALGORITHM}

In this section, we will give an exact algorithm for detecting all Global Spatial Clusters in $\mathbb{R}^{2}$ space. The basic idea is to transform the input spatial space from Cartesian coordinate system to polar coordinate system, and based on which an angular sweep procedure is repeatedly invoked for each node to ensure that no GSC is missed.

\subsection{Local Spatial Cluster}

Global Spatial Cluster is defined based on covering circle with fixed radius, and the following will give the definition of a more restricted covering circle, called $v$-bound circle, and based on which Local Spatial Cluster (LSC) is defined.

Definition 5 ( $v$-BOUNDED CIRCLE). Given a point $v \in \mathbb{R}^{2}$, if $a$ circle with diameter d (user-specified distance threshold) passes $v$, then it is called a v-bounded circle denoted as $C_{v}$.

For a given point $v$, set it as reference point and $x$-axis as reference direction to build a polar coordinate system. If the center of a $v$-bounded circle $C_{v}$ has coordinate $(r, \theta)$ in this polar coordinate system, denote this circle as $C_{v}(r, \theta)^{1}$ where $\theta \in\left(-180^{\circ}, 180^{\circ}\right]$. Now we give the definition of $v$-Local Spatial Cluster as follows.

Definition 6 ( $v$-Local Spatial Cluster (LSC)). Let $r=d / 2, a$ $v$-Local Spatial Cluster $L_{v}$ is a set of points enclosed by circle $C_{v}(r, \theta)$ such that there does not exist a circle $C_{v}\left(r, \theta^{\prime}\right)$ enclosing a proper superset of $L_{v}$. Denote the set of all $v-L S C s$ for a fixed $v$ as $\mathcal{L}_{v}$.

We then have the following lemma showing the relationship between global and local spatial clusters, which is the backbone of the exact spatial algorithm.

LEMma 1. Given a set of points $V$ in $\mathbb{R}^{2}$ and a distance threshold, denote the set of all GSCs as $\mathcal{G}$, then $\mathcal{G} \subseteq \cup_{v \in V} \mathcal{L}_{v}$.

Proof. As shown in Fig. 2, let all the small circles consist a GSC $G$, by definition, there is a circle with radius $r=d / 2$ covering them (shown as the large black circle). Let $e d(u, v)$ denote the spatial distance (Euclidean distance) between two users. W.o.l.g., assume that $a$ is the farthest point in the circle from $O$ and then the dashed circle centered at $O$ with radius $r^{\prime}=e d(a, O)<r$ still encloses all points in $G$. Find a point $Q$ on the line $\overrightarrow{a O}$ such that $e d(a, Q)=r$ and get the grey circle centered at $Q$ with radius $r$ shown as the grey circle. For any point $b \in G$, based on triangle inequality, $e d(b, Q) \leq e d(b, O)+r-r^{\prime} \leq r$. Thus all points in $G$ can be covered by the $a$-bounded grey circle, i.e., $G$ is an $a$-LSC.

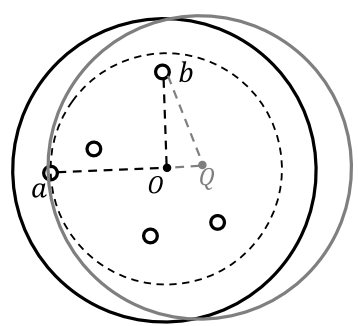

Figure 2: Illustration of Lemma 1.

\subsection{Searching All LSCs}

Lemma 1 shows that the problem of finding all GSCs can be solved by calculating $v$-LSCs $\mathcal{L}_{v}$ for every $v \in V$. To efficiently calculate $\mathcal{L}_{v}$ for a given reference point $v$, in this subsection, we introduce the Angular Sweep-based technique.

Suppose that circle $C_{v}(r, \theta)$ rotates counterclockwise, i.e., $\theta$ increases from $-180^{\circ}$ to $180^{\circ}$, for each point within distance $d=2 r$

${ }^{1}$ We alternatively use $C_{v}(r, \theta)$ and $C_{v}$ if the context is clear. 


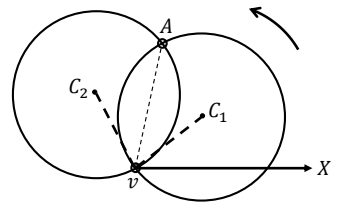

Figure 3: two events for $A$.

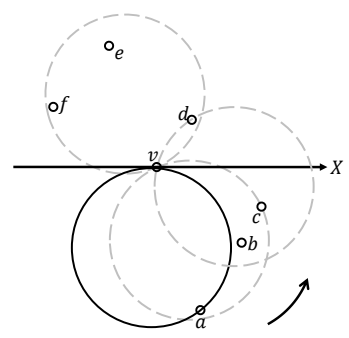

Figure 4: Angular Sweep

from $v$, we consider two special events: it first enters $C_{v}$ and it quits $C_{v}$, and we call the angles $\theta$ at these two special events as start angle $\theta$.start and end angle $\theta$.end respectively. When $\theta \in[\theta$.start, $\theta$.end $]$, the circle $C_{v}(r, \theta)$ always encloses this point. Figure 3 illustrates such rotation process.

In Figure 3 , the circles centered at $C_{1}$ and $C_{2}$ are $C_{v}$ at the two special events for point $A$. Denote polar coordinates of $C_{1}$ and $C_{2}$ as $(r, \theta$.start $)$ and $(r, \theta$.end $)$ respectively, and polar coordinate of $A$ as $\left(d_{A}, \alpha_{A}\right)$, then we calculate $\theta$.start and $\theta_{\text {.end }}$ using equations:

$$
\begin{gathered}
\theta . \text { start }=\alpha_{A}-\cos ^{-1} \frac{d_{A}}{2 r} \\
\theta . \text { end }=\alpha_{A}+\cos ^{-1} \frac{d_{A}}{2 r}
\end{gathered}
$$

Given a reference point $v$ and a set of vertexes $V^{\prime} \subset V$ where each vertex is within $d$ from $v$, Algorithm 3 outputs all $v$-Local Spatial Clusters. Lines 1 - 5 first calculate start and end angles for nodes in $V^{\prime}$ via Eq. (1) and Eq. (2) and sort nodes based on start angles. Lines 6 - 19 present the angular sweep procedure (Fig. 4).

Let the initial state of $C_{v}(r, \theta)$ (shown as the black circle in Fig. 4) be at the place where it just passes the first node $(\theta=a$.start $)$ and let the candidate set $C S=\{a\}$ which records the set of points currently enclosed by $C_{v}$. Let end keep track of the smallest end angle of points in $C S$. Keep rotating $C_{v}$ counter-clockwisely to the next points and adding new points to $C S$ until one point in $C S$ will leave $C_{v}$. More specifically, denote the next point that $C_{v}$ is going to reach as $x$, when $x$.start $>$ end which means that at least a point is going to leave $C_{v}$, add $C S$ to LSC set. Rotate $C_{v}$ to reach $x$, add $x$ to $C S$ and remove points whose end angles are less than $x$.start to form a new candidate set. Keep the above procedure until reaching the last point. For example, in Fig. 4, add $b, c$ to $C S$ step by step and then when $C_{v}$ is going to enclose $d$, since $d$.start $>$ end $=$ a.end, the current set $C S=\{a, b, c\}$ should be a LSC. Then remove point $a$ from $C S$ because it has left $C_{v}$ and add $d$ to $C S$. Keep rotating and generating LSC until the circle encloses the last point $f$. There are three LSCs detected as the grey dashed circles enclose.

Complexity Analysis. Suppose that the input vertex set $V^{\prime}$ has a size $m$, then Line 5 takes time $O(m \log m)$ by using a conventional sorting algorithm. For the angular sweep shown in lines 6-19, the update of candidate set $C S$ (lines 13 to 15), which dominates the loop body, is executed in $O(m)$ time. Thus, the total worst case time complexity of Algorithm 3 should be $O\left(m \log m+m^{2}\right)=O\left(m^{2}\right)$. For any vertex $v$, the number of $v$-LSCs is $O(m)$.

\subsection{Searching GSC}

An LSC may not be a GSC as it might be a subset of another LSC with a different reference node. Thus, by excluding any LSC which is a subset of another LSC, we obtain all GSCs.

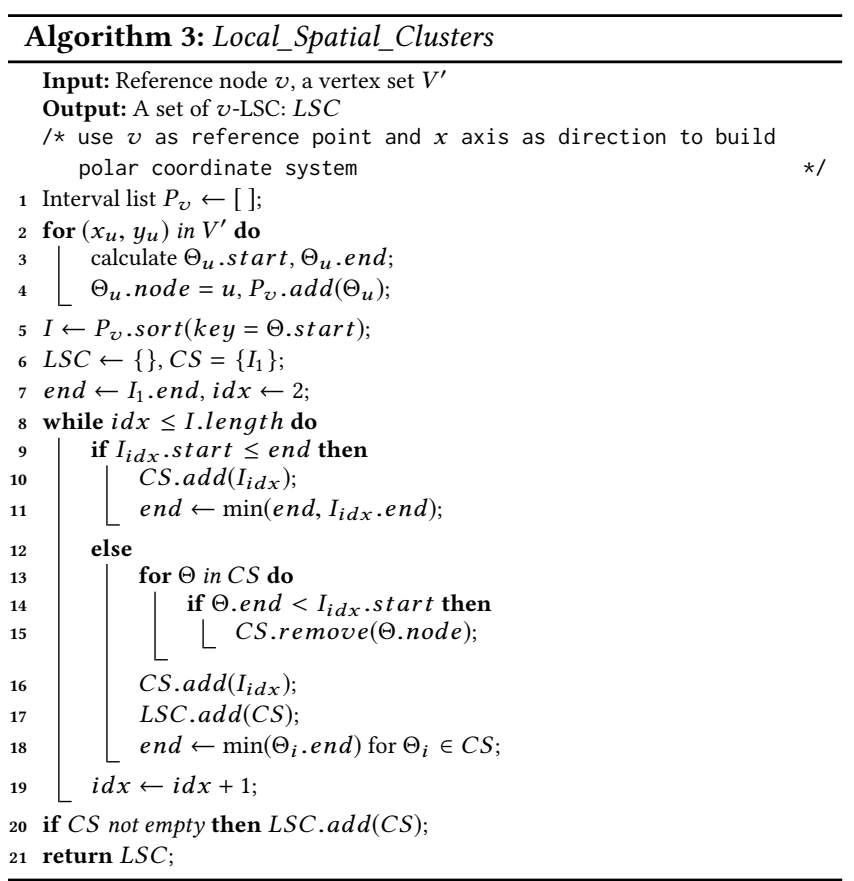

The whole algorithm to find GSCs is presented in Algorithm 4. Note that for a certain social constraint, e.g., $k$-core, $k$-truss, some simple pruning can be implemented to reduce search space. Algorithm 4 uses $k$-core as an illustration. In the experiments, we implement both $k$-core and $k$-truss. For each node $v$, to reduce search space, line 3 applies range query to find out vertexes within distance $d$ from the location of $v$ since any vertex outside this circle can not be in a $v$-LSC. Since we need to find $k$-core at last, if the number of vertexes lie in the circle is less than $k$, these LSCs can not contain any MCC and we skip them as line 4 shows. Line 5 invokes Algorithm 3 LocalMaximalSet to find out all $v$-LSCs. After detecting all LSCs, the function FindGSC is invoked to add LSCs which are not subset of any others to the GSCs set GSC.

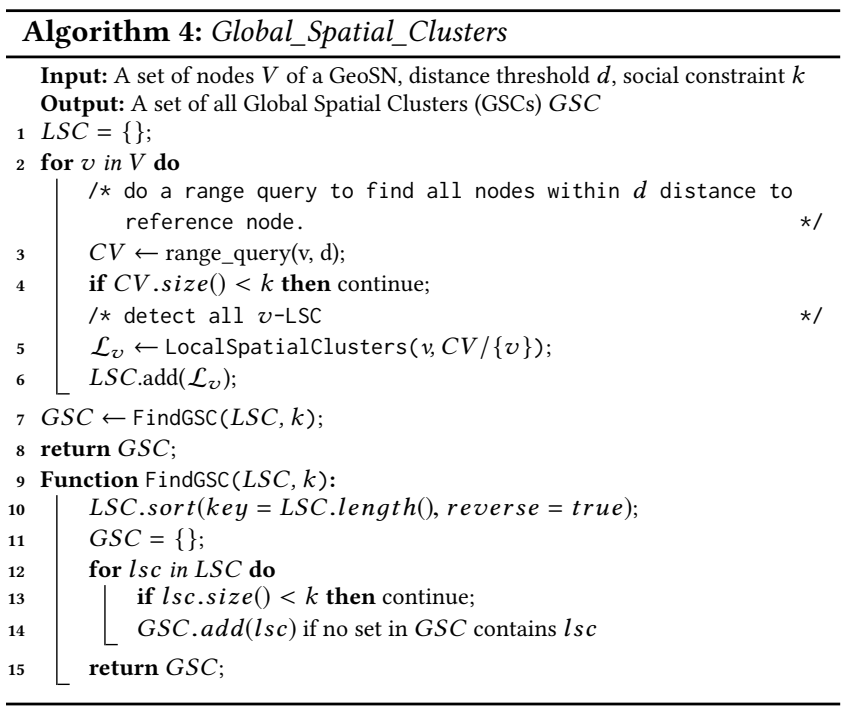


Complexity Analysis. Assume that there are $n$ vertexes in GeoSN, i.e., $|V|=n$, in the worst scenario, for each vertex $v \in V$, there are $O(n)$ vertexes within distance $d$ to $v$, and thus the worst time complexity for finding $v$-LSCs (line 5) would be $O\left(n^{2}\right)$ as analyzed in last subsection. Thus, finding all LSCs would cost $O\left(n^{3}\right)$. There are $O\left(n^{2}\right)$ LSCs in total, thus function FindGSC will do $O\left(n^{4}\right)$ set comparisons where each single comparison takes time $O(n)$. The total time complexity in the worst case should be $O\left(n^{3}+n^{5}\right)=O\left(n^{5}\right)$. However, in practice, the spatial threshold $d$ is a small value. Assume that the location density of points is $\rho$, and let $C_{x}=\rho \pi(x / 2)^{2}$, then it takes time $O\left(C_{2 d}^{2}\right)$ to get $v$-LSCs, the number of $v$-LSCs would be $O\left(C_{2 d}\right)$ and each set has points $O\left(C_{d}\right)$, so the time complexity would be $O\left(n C_{2 d}^{2}+n^{2} C_{2 d}^{2} C_{d}\right)=O\left(n^{2} C_{2 d}^{2} C_{d}\right)$.

\section{PRUNING AND OPTIMIZATION}

The high time complexity of Algorithm 4 in last section prevents it being scaled to large dataset. Thus, we propose several pruning strategies and optimization tricks for Algorithm 4, which is experimentally demonstrated to accelerate the algorithm a lot and reduce time by orders of magnitude in some datasets.

In Algorithm 4, in the worst case an LSC needs to be compared with other $O\left(n^{2}\right)$ LSCs to determine whether or not it is a GSC, which is extremely inefficient and is the dominant part of the time complexity. In this section, we develop pruning rules to dramatically reduce the times of set comparisons.

Pruning rule I: point-wise pruning. Given an $a$-LSC and a $b$-LSC ( $a$ is a different point from $b$ ), a trivial observation is that if $e d(a, b)>d$, one of them can never be a superset of the other and there is no need to perform element-wise set comparison.

However, in many situations, even though ed $(a, b)$ is smaller than $d$, it is very likely that an $a$-LSC can never cover a $b$-LSC, as Fig. 5 (b) shows. The following will seek a stronger pruning rule in the granularity of LSCs so that we only need to check elements of two LSCs when necessary.

Assume that there is a set of points $S$ and there exists a circle $C$ with radius $r$ covering all points in $S$, and we now consider the problem to decide the location of $C$. Denote the circle center of $C$ as $C_{o}$, for any point $s \in S$, we have $e d\left(s, C_{o}\right) \leq r$. We draw a circle with radius $r$ centered at each point in $S$, then $C_{o}$ must lie in the intersection of these circles. We relax these circles with their minimum bounding rectangles, and $C_{o}$ must lie in the intersection area of these rectangles. The intersection rectangle is trivial to compute: instead of considering all points in $S$, we only need four values: $x_{\max }, x_{\min }, y_{\max }$ and $y_{\min }$, which are the maximal and minimal $x$ coordinates and $y$ coordinates of points in $S$ respectively. As Fig. 5 (a) shows, there are three points filled with grey that decide the intersection rectangle. The dashed rectangle centered at the uppermost or rightmost point decide the bottom side or left side of intersection rectangle respectively, while the one centered at the leftmost and also bottom-most point decide the right and upper side of intersection. The rectangle is thus calculated by CenterRec $=$ $\left\{(x, y) \mid x \in\left[x_{\max }-r, x_{\min }+r\right], y \in\left[y_{\max }-r, y_{\min }+r\right]\right\}$.

For two LSCs with different reference nodes, we consider the necessary condition for a set to cover another. As Fig. 5 (b) show, there are two bounded circles with threshold $d$ as diameter, shown as black and grey large circles, covering an $a$-LSC and $b$-LSC respectively. For each LSC, we calculate the rectangle CenterRec as the

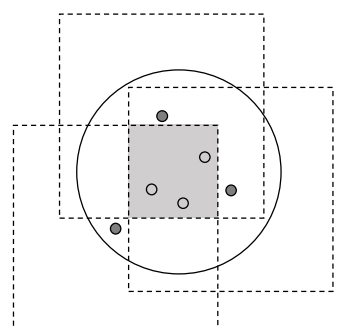

(a)

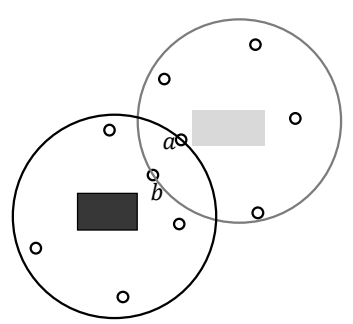

(b)
Figure 5: Illustration of pruning rules.

black and grey shadows show respectively. Since their CenterRecs do not intersect with each other, it is not likely to find a circle with diameter $d$ to cover all points in these two sets, thus neither of the two LSCs can cover the other. The following is a stricter pruning rule,

Pruning rule II: LSC-wise pruning. Given an LSC $S$, we only need to do set comparison between $S$ and each of those LSCs whose CenterRec intersect with that of $S$.

Implementation. By applying these two pruning rules, we re-implement the function FindGSC in Algorithm 4, called FindGSCPrune. As Algorithm 5 shows, for any point $v$, the point-wise pruning rule is first applied. Nearby candidate points points is found by using a range query, and then a set of all LSCs with reference node in points are gathered for comparison (cprSets in Algorithm 5). To further reduce set comparisons, for each $v$-LSC $s$, set-wise pruning rule is applied so that we only compare $s$ with sets in cprSets each of which has a CenterRec intersecting with s's.

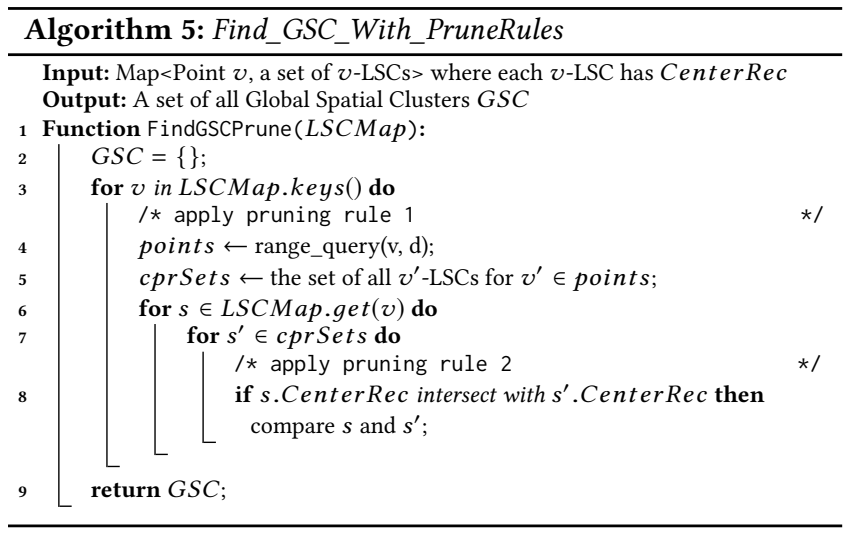

\section{APPROXIMATE SPATIAL ALGORITHM}

In last section, we propose powerful pruning rules, though it works in practice, it would still be desirable to pursue a more scalable algorithm for large scale GeoSN. In addition, in the exact algorithm, only after all LSCs are detected can we decide if an LSC is global. However, in many scenarios, users would expect to get GSCs in a more interactive way, i.e., we should return some GSCs before all LSCs are detected. In this section, we will show that if we loose the spatial constraint, then a much more efficient and interactive algorithm with constant approximation ratio $(\sqrt{2})$ can be designed.

\subsection{The Basic Intuition}

In Fig. 6, assume that the small black points consists of a Global Spatial Cluster, then based on the definition, there is a circle, shown 


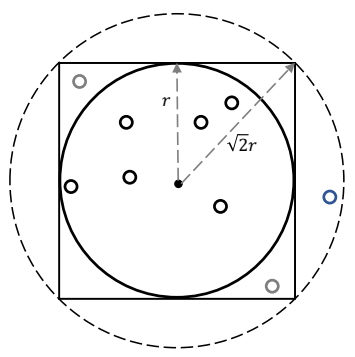

Figure 6: Illustration for theorem 1.

as the large black circle, with diameter $d$ which is the spatial distance threshold to cover this cluster. We relax this circle by its minimum bounding rectangle, shown as the black rectangle in the figure, and this rectangle must cover all points in that cluster. Similar to the definition of GSC, we give that of Global Approximate Spatial Cluster (GASC) based on rectangles.

Definition 7 (Global Approximate Spatial Cluster). Given a set of points $U \subset V$ and a distance threshold $d, U$ is a Global Approximate Spatial Cluster if,

- there exists a rectangle $R$ with side length d covering $U$;

- there does not exist a rectangle with side length d covering a set of points $U^{\prime}$ such that $U \subsetneq U^{\prime}$.

The rectangle $R$ covering $U$ is called a global maximal square.

We give a theoretical bound for using GASCs to replace GSCs,

Theorem 1 (Sandwich Theorem). For a distance threshold $d$, denote the set of all GSCs as $\mathcal{U}_{d}$ and the set of all GASCs as $\mathcal{A}_{d}$. Then we have the following theorem,

- For any set $U \in \mathcal{U}_{d}, \exists A \in \mathcal{A}_{d}$ such that $U \subset A$.

- For any set $A \in \mathcal{A}_{d}, \exists U^{\prime} \in \mathcal{U}_{\sqrt{2} d}$ such that $A \subset U^{\prime}$.

Proof. Fig. 6 illustrates this lemma. The first property is trivial. For the second property, let the black rectangle denote a global maximal square covering an GASC, then its minimum bounding circle, denoted as the black dashed circle, with radius $\sqrt{2} d / 2$ must cover this GASC.

Based on this theorem, the problem of detecting all GSCs can be approximated by finding all GASCs with approximation ratio $\sqrt{2}$. Similar to $v$-bounded circle and $v$-LSC, we give the definitions of square with $x$-bounded left side (with shorthand as $x$-bounded square) and $x$-Local Approximate Spatial Cluster ( $x$-LASC) as follows,

Definition 8 (SouARe With $x$-Bounded Left Side). Given $a$ square with side length $d$, it is a square with $x$-bounded left side if the left side of this square passes node $x$.

Definition 9 (Local Approximate Spatial Cluster). Given an $x$-bounded square $R_{x}$ and a set of nodes $U_{x}$ covered by $R_{x}, U_{x}$ is a $x$-Local Approximate Spatial Cluster $(x-L A S C)$ if and only if $U_{x} \neq \emptyset$ and there does not exist a set of nodes $U_{x}^{\prime} \supsetneq U_{x}$ covered by another $x$-bounded square. Denote the set of all $x$-LASC for a fix $x$ as $\mathcal{U}_{x}$.

Similar to Lemma 1, we have the following lemma showing the relationship between GASCs and LASCs.

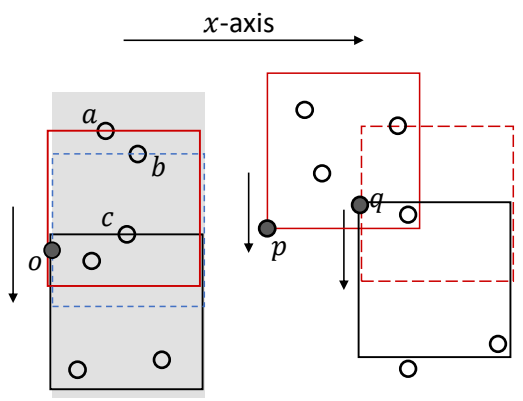

Figure 7: Illustration of Algorithm 6

LEMmA 2. Given a set of points $V$ in $\mathbb{R}^{2}$ and a distance threshold $d$, denote the set of all Global Approximate Spatial Clusters as $\mathcal{U}$. It always holds that $\mathcal{U} \subset \cup_{x \in V} \mathcal{U}_{x}$.

Based on Lemma 2, the problem of finding all GASCs can be transformed to finding LASCs as candidates and then generating GASCs from the candidate set.

\subsection{Algorithm}

Algorithm 6 presents the whole procedure to detect all GASCs interactively by a single scan of all nodes. Line 2 first sorts points by $x$-coordinates. For each point, it generates all LASCs and calls function CheckGlobal to check if each LASC is a global ASC. The following explains the detail of these two procedures.

Detecting LASCs (lines 4-13). Fig. 7 illustrates the steps of finding LASC. For point $O$ with coordinate $(O . x, O . y)$, the possible points that a $O$-bounded square can cover is in the rectangle $\{(x, y) \mid x \in[O . x, O . x+d], y \in[O . y-d, O . y+d]\}$ as the grey shadow rectangle shows. Then we generate all $O$-LASCs by moving a $d \times d$ rectangle downwards in the shadow. The points in the grey shadow are sorted by $y$ coordinates, and then let the upper side of an $O$ bounded square pass the points one by one. Let start keeps tracking of the first point that has not been covered and $C S$ denote currently covered. Initially, let the upper side of rectangle passes the first point $a$ (line 6). Get all points covered by this rectangle (lines 12 - 13) and this should be a LASC since when the rectangle moves downwards, it can not cover $a$ anymore. Move it downwards so that its upper side passes the next point, and there can be three possible situations: if the last point in the grey shadow has been enclosed by the previous rectangle, stop moving and terminate (line 9); if the new rectangle does not enclose any new node, then ignore it (line 10); if the new rectangle encloses more points than the previous one, then get all nodes covered by this square and it is a LASC (lines 11-13). For example, in Fig. 7, when the rectangle moves to pass the second point $b$, no new points is covered in the blue dashed rectangle and thus it is skipped, while when it passes $c$, new points are included and all points enclosed forms a LASC. Since the last point has been covered, it terminates.

Finding GASCs (lines 19-25). Once an LASC is found, it is easy to check if this is a GASC. For example, in Fig. 7, there is a $q$-LASC covered by the red dashed rectangle. To check if this is a GASC, we only need to compare it with $p$-LASCs where $p . x<$ $q . x$ which have already been detected, since any $q^{\prime}$-LASC where $q^{\prime} . x>q . x$ can not contain point $q$. Only at most three points in the dashed rectangle needed to be considered. The three points 
are: point with maximum $x$ coordinate and points with minimum and maximum $y$ coordinates. If these three points are already in a previous LASC, then all points in the rectangle are in it, thus this LASC will be discarded. Otherwise, this is an GASC. Function CheckGlobal of Algorithm 6 shows this process where NodeGASC records for each point a set of all GASCs currently found that enclose it. By determining if the three GASCs sets for these three special points have intersection, we can check if the LASC is a global one.

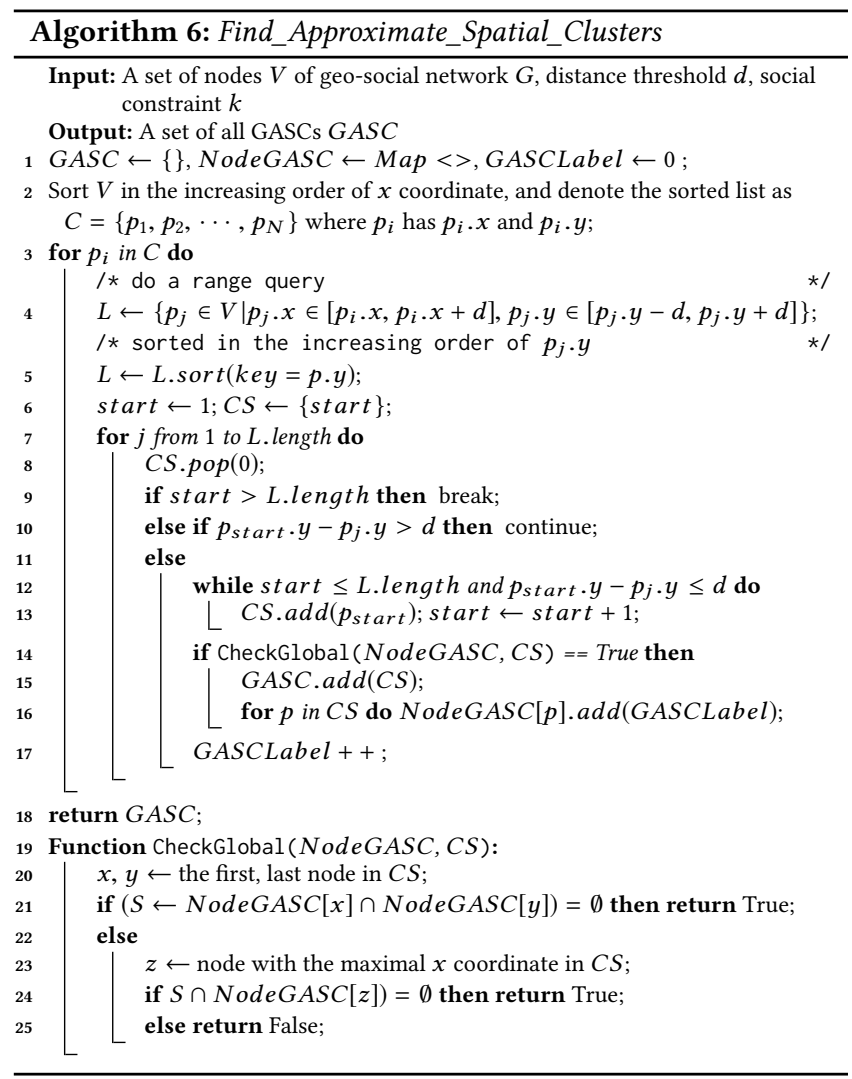

Complexity Analysis The average number of points in a $d \times 2 d$ rectangle is $C=2 \rho d^{2}$. For each point $v \in V$, lines 7 to 13 take time $O(C)$ to compute all $v$-LASCs. For function CheckGlobal, the dominate step is set intersections. Suppose there are $O(D)$ GASCs that may contain a point $x$, i.e., the size of NodeGASC $[x]$ is $O(D)$, then conducting a set intersection operation would take $O(D)$, then the total time complexity is $O(n \log n+n \times(C \times D))=O(n \log n+$ $n C D)$. In the worst case, $D=O\left(C^{2}\right)$, however, since NodeGASC $[x]$ records only GASCs currently found instead of all LASCs that contain $x, D$ is practically very small.

\section{EXPERIMENTAL STUDIES}

Our experiments contain three parts: we first test and compare the spatial algorithms which find out all spatial clusters, then test the whole MCC framework to get all maximal co-located communities, and finally we conduct case studies to compare our results with two state-of-the-art researches. All of our algorithms are implemented by Java using JDK 11 and tested on an Ubuntu server with Intel(R) Xeon(R) CPU X5675 @ 3.07GHz and 64 GB memory.

\subsection{Spatial Algorithm Evaluation}

In this part, we test the performance of our spatial algorithms and compare with the state-of-the-art algorithm from [3, 23].

Algorithms We test four algorithms as shown in Table. 2. The clique-based algorithm from [3] does not solve exactly the same problem as ours, however, it can be easily adapted to detect all spatial clusters by enumerating all cliques of a spatial graph.

Table 2: List of implemented algorithms.

\begin{tabular}{cc}
\hline Name & Algorithm \\
\hline clique & adapt clique-based algorithm [3] to get all spatial clusters \\
exact + rule 1 & algorithm 4 with pruning rule 1 \\
exact + rule 1,2 & algorithm 4 with pruning rule 1 and 2 \\
approx & approximation algorithm Algorithm 6 \\
\hline
\end{tabular}

Dataset The experiments are conducted on three real-world datasets and two synthetic datasets. Table. 3 presents the statistics of the spatial part of three real geo-social networks. \#Neighbors is defined as the number of people within 500 meters from a specific user and we calculate the average and maximum number of \#Neighbors. The locality level is defined as the ratio between max. \#Neighbors and avg. \#Neighbors. For example, for the weibo dataset, the max. \#Neighbors is high while avg. \#Neighbors is low, so it has relatively high spatial locality.

Table 3: Statistics of real-world spatial datasets.

\begin{tabular}{ccccc}
\hline Dataset & Size (1K) & $\begin{array}{c}\text { Max. } \\
\text { \#Neighbors }\end{array}$ & $\begin{array}{c}\text { Avg. } \\
\text { \#Neighbors }\end{array}$ & Locality \\
\hline Brightkite [5] & 51 & 1342 & 55.67 & medium \\
Gowalla [5] & 107 & 536 & 15.38 & low \\
Weibo[16] & 1,020 & 976 & 15.85 & high \\
\hline
\end{tabular}

Parameter Settings Table. 4 shows the parameter settings for both synthetic and real datasets. For the synthetic datasets, there are three parameters: the number of points $N$, density and distance threshold $d$. For real datasets, we consider two parameters: the percentage of users sampled from the original datasets ratio and distance threshold $d$. At each time, we vary one parameter while other parameters are set to their underlined default values.

Table 4: Table of parameter setting.

\begin{tabular}{ccc}
\hline Category & Parameter & Values \\
\hline \multirow{3}{*}{ synthetic } & $N$ & {$[50,100,200, \underline{300}, 400,500,1000] \times 1 K$} \\
& density & {$[0.001,0.002,0.004,0.008,0.012,0.02]$} \\
& $d$ & {$[5,15, \underline{30}, 45,60]$} \\
\hline \multirow{2}{*}{ real } & ratio & {$[0.2,0.4,0.6,0.8, \underline{1.0}]$} \\
& $d$ & {$[100,300, \underline{500}, 700,1000]$} \\
\hline
\end{tabular}

6.1.1 Scalability. To test the scalability of our algorithms, we vary $N$ for synthetic datasets and ratio for real datasets. The results are shown in Fig. 8(a)-8(e). The clique-based algorithm increases exponentially as the number of points increases on all datasets, which demonstrates the NP-hardness nature of the clique enumeration problem. Our exact algorithm significantly outperforms cliquebased algorithm: 1) on synthetic datasets, it outperforms clique by one to two orders of magnitudes and clique can not terminate in $8,000 \mathrm{~s}$ for more than $500 \mathrm{~K}$ data points while exact can return results in 100 seconds. 2) on real datasets, our algorithm outperforms 


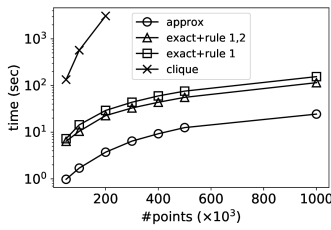

(a) time vs. $N$ (Uniform)

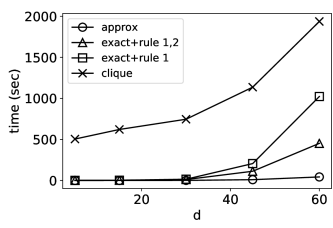

(f) time vs. $d$ (Uniform)

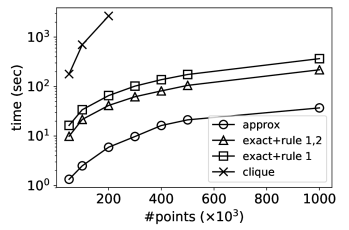

(b) time vs. $N$ (Gaussian)

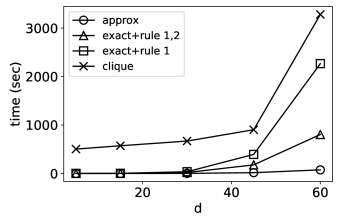

(g) time vs. $d$ (Gaussian)

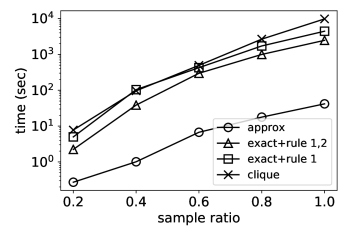

(c) time vs. ratio (Brightkite)

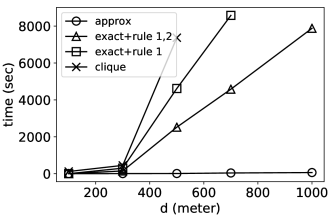

(h) time vs. $d$ (Brightkite)

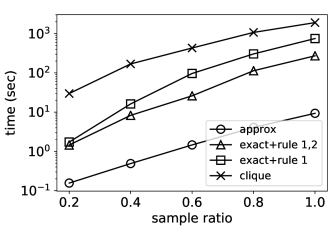

(d) time vs. ratio (Gowalla)

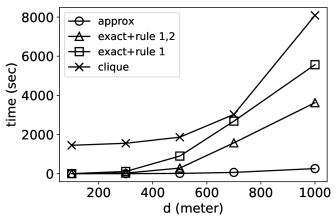

(i) time vs. $d$ (Gowalla)

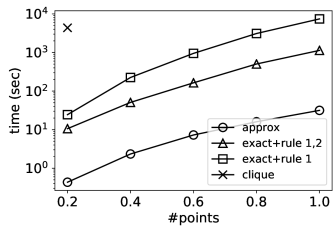

(e) time vs. ratio (Weibo)

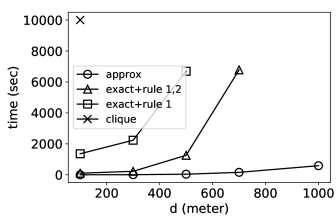

(j) time vs. $d$ (Weibo)

Figure 8: Experiment results of the influence of scalability and $d$ on synthetic and real-world datasets.

clique especially for large-sized Weibo dataset where clique can not return results in $8,000 \mathrm{~s}$ when sampling $40 \%$ data points. Our exact and approximation algorithms show strong scalability on synthetic datasets, since they show near-linear increase when the number of points increases. Notably, on three real datasets, the increase is faster than that on synthetic data since when the ratio becomes larger, not only the number of data points, but also the density increase.

6.1.2 Effect of $d$. Fig. 8(f)-8(j) present the execution time by varying the distance threshold $d$. For clique, when $d$ increases, the execution time increases dramatically, e.g., it can not return results when $d=700$ meters for Brightkite and weibo datasets. For exact algorithms, when implemented with both two pruning rules, the execution time is much less than that of using only one pruning rule and it becomes more obvious when $d$ increases. The execution time of approx does not show obvious change w.r.t. $d$ comparing to other algorithms. For the weibo dataset, when $d$ is set as 700 or 1000 meters, approx still return results in short time while other three algorithms cannot terminate within $8,000 \mathrm{~s}$.

We briefly give the reason here. For clique, when $d$ increases, the virtual spatial neighborhood network would be more complex and thus enumerating all maximal cliques would be much more time-consuming. For the exact algorithm, as $d$ increases, the number of LSCs and the number of points in each LSC increase, and accordingly the time spent on set comparisons for LSCs would be a major bottleneck. Recall that the time complexity for the exact algorithm has $T \propto n^{2} d^{5}$. Pruning rule 1 decreases the times of set comparisons by excluding comparisons between two LSCs with reference nodes distance larger than $d$. When $d$ increases, the percentage of set comparisons pruned by this rule would decrease and thus it loses the pruning power. However, for pruning rule 2, it is still very effective when $d$ grows since it is a set-wise pruning method instead of point-wise.

6.1.3 Effect of data density. Fig.9 shows the execution time w.r.t. different densities of synthetic datasets. As density increases, the execution time of both clique and exact+rule1 increases quickly, however, when implemented with both pruning rules, the exact algorithm grows much more slowly. The effectiveness of pruning rule
II becomes more obvious with the increase of density. For Gaussian distributed datasets, which have higher locality than uniform data, the pruning rule II reduces more than $50 \%$ execution time than exact+rule1 when density is set as 0.02 . Density affects exact algorithms due to the same reason as $d$ does, both of them increase the number and set size of LSCs, which makes LSCs comparisons more costly. The execution time of approximation algorithm increases very slowly since there are only at most three set comparisons needed to be conducted for each local approximate spatial cluster even though density changes.

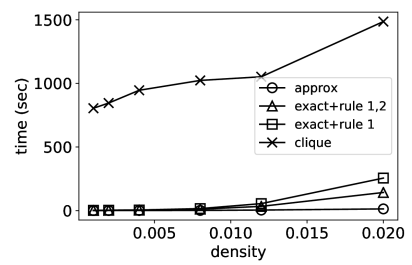

(a) time vs. density (Uniform)

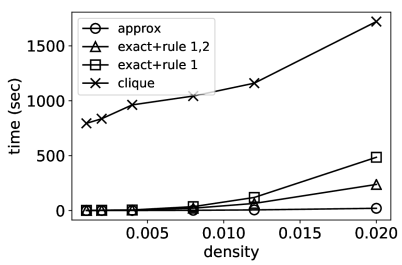

(b) time vs. density (Gaussian)
Figure 9: Experiment results of the influence of density.

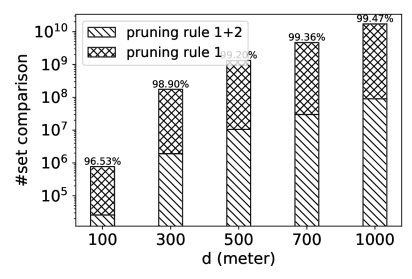

(a) when $d$ varies (Gowalla)

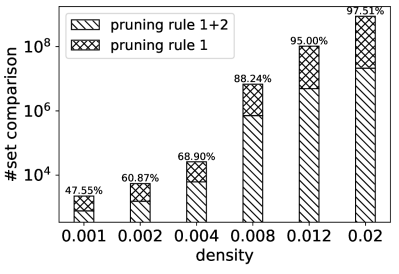

(b) when density varies (Uniform)
Figure 10: The effectiveness of pruning rule 2.

6.1.4 Effectiveness of pruning rules. As we have analyzed before, the bottleneck of time complexity for exact algorithms is set comparisons for local spatial clusters and the two pruning rules decrease time by reducing set comparisons at different levels. As we have shown, when $d$ or density increase, the pruning rule 2 become more effective in reducing execution time. To further present the 
effectiveness of different pruning rules, we record the numbers of set comparisons when implementing only first pruning rule or both rules. Fig. 10 shows the results on Gowalla and uniform synthetic datasets. Pruning rule 2 can help decrease the number of set comparisons by orders and when $d$ or density increase, it is observed to reduce more set comparisons. When $d$ is set as $1 \mathrm{~km}$ on Gowalla dataset, pruning rule 2 can reduce more than $99 \%$ set comparisons of exact+rule1.

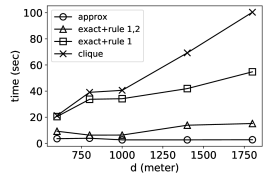

(a) time vs. $d$ (Brightkite)

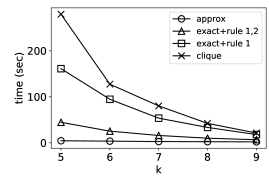

(d) time vs. $k$ (Brightkite)

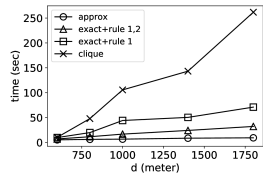

(b) time vs. $d$ (Gowalla)

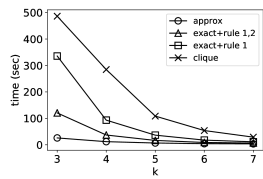

(e) time vs. $k$ (Gowalla)

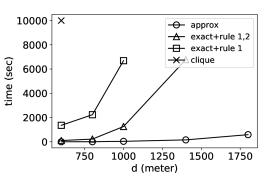

(c) time vs. $d$ (Weibo)

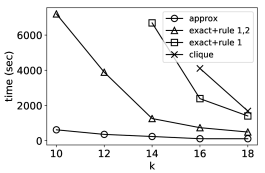

(f) time vs. $k$ (Weibo)
Figure 11: MCC framework evaluation results of the influence of $k$ and $d$ on real geo-social networks.

\subsection{Framework Evaluation}

The previous subsection presents the results for spatial algorithm and this part will demonstrate the efficiency and effectiveness for the whole framework to detect all maximal co-located communities, which is shown in Algorithm 1. The experiments are conducted on three real world geo-social networks and Table 5 shows the statistics of social network information. Note that before running the algorithms, we do some data cleaning works for original datasets, e.g., deleting all self-loop edges.

Table 5: Statistics of social network information of realworld datasets.

\begin{tabular}{ccccc}
\hline Dataset & \#Vertices & \#Edges & Avg. Degree & Max Degree \\
\hline Brightkite & $58 \mathrm{~K}$ & $214 \mathrm{~K}$ & 7 & 1134 \\
Gowalla & $69 \mathrm{~K}$ & $175 \mathrm{~K}$ & 5 & 739 \\
Weibo & $1,019 \mathrm{~K}$ & $8,245 \mathrm{~K}$ & 16 & 1100 \\
\hline
\end{tabular}

Table 6: Parameter setting for framework.

\begin{tabular}{ccc}
\hline Dataset & $k$ values & $d$ values (meter) \\
\hline Brightkite & {$[5,6, \underline{7}, 8,9]$} & {$[600,800, \underline{1000}, 1400,1800]$} \\
Gowalla & {$[3,4, \underline{5}, 6,7]$} & {$[1000,1250, \underline{1500}, 1750,2000]$} \\
Weibo & {$[10,12, \underline{14}, 16,18]$} & {$[1000,1250, \underline{1500}, 1750,2000]$} \\
\hline
\end{tabular}

For social constraint in the framework, we implement both $k$ core and $k$-truss, however, due to the limit of page, we only present the evaluation results of framework based on $k$-core, and the results on $k$-truss have very similar performance. To make the framework more efficient, we adopt a simple pruning rule similar to the one used in [3]. The pruning rule is based on the fact that a MCC must be a subset of a $k$-core (or $k$-truss), thus we first generate all $k$-cores from social network by applying core decomposition algorithm, and then apply our framework in each $k$-core to get all MCCs. Table 6 shows the settings of two parameters: $k$ (of $k$-core) and distance threshold $d$.

6.2.1 Effect of $d$. Fig. 11(a)-11(c) show the total execution time w.r.t. $d$. Since we apply spatial algorithm in each $k$-core instead of for all data points, the execution time for detecting MCCs is much less than that of detecting all spatial clusters presented in the last subsection. Clique is the slowest one on all datasets and increases dramatically w.r.t. $d$. The exact algorithms present efficiency on Brightkite and Gowalla datasets and the time does not increase much as $d$ increases. However, for Weibo dataset, time increases quickly with $d$. A possible reason is that data points in Weibo have much higher degree and there can be a $k$-core consisting of many data points, and applying the spatial algorithm in that core can still be time consuming and the change of time w.r.t. $d$ is similar to the spatial algorithm experiment result as Fig. 8(j) shows.

6.2.2 Effect of $k$. Fig. 11(d)-11(f) present results on three real datasets by changing $k$. The execution time for all datasets decreases dramatically when $k$ turns larger. The reason is that, when $k$ increases, each $k$-core on social network would have smaller size, thus applying our framework on each $k$-core would save time.

6.2.3 Correctness of approximation algorithm. The above results have already demonstrated the efficiency and scalability of our approximation algorithm. To further validate its correctness, in each community detected by applying the approximation algorithm as spatial algorithm, we calculate the maximum pairwise distance as the community distance and Fig.12 present the average and maximum community distance of all communities. It shows that the community distance is always bounded by $\sqrt{2} \cot d$ and the average distance is normally smaller than $d$ which means many communities have distance smaller than exact threshold.
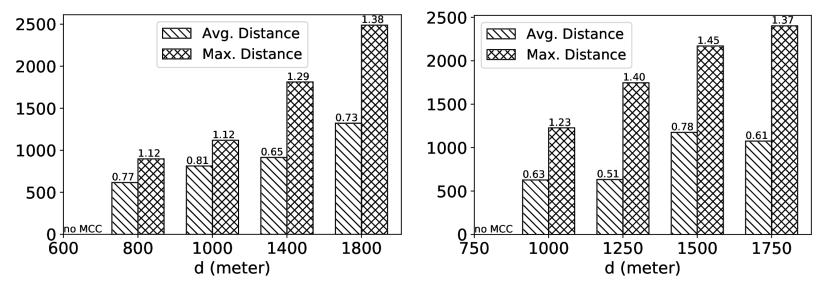

Figure 12: Distance of MCCs on Brightkite and Gowalla.

\subsection{Case Studies}

We implement the algorithms in $[3,11]$. Both the two papers have different problem definitions with us. [11] provides a community search algorithm where the distance constraint is not defined in the same way as our work, and [3] solves the problem to find only the maximum MCC and it applies all-pair distance constraint as spatial constraint. We conduct two case studies on Gowalla and Brightkite datasets by using our approximation algorithm and compare the result with that of $[3,11]$ respectively to demonstrate the effectiveness of our problem and algorithm.

6.3.1 Bounded Spatial Distance Guarantee. We conduct experiment on Gowalla dataset and set $k=2$ and $d=2 \mathrm{~km}$. Fig. 13 (a) shows all MCCs detected by our algorithm in a small region. Each circle is the location of the MCC center and the color indicates the number 
of community members. There are 20 MCCs in this region. We also present two communities shown as the red circles in (b) and (c) respectively retrieved by using the community search algorithm in [11] with two different query users. In (b), the purple circle with diameter $d$ covers a MCC found by our algorithm. The method in [11] only returns a small subset of our MCC in order to make sure that the covering circle has the minimum radius. In (c), [11] returns a community that has a minimum covering circle with diameter much larger than $d=2 \mathrm{~km}$, and is not detected as a MCC by our algorithm. As Fig.13 shows, [11] does not allow user to specify the distance threshold, and different MCCs do not have consistent distance bound. For a query user who have many nearby friends, [11] may return a small subset, however, for user who do not have nearby friends, it still returns a cluster with large distance among cluster members.

6.3.2 Diverse MCCs. On Brightkit dataset, by setting $d=1 \mathrm{~km}$ and $k=4$, we detect 32 MCCs. We conduct hierarchical cluster analysis on 32 sets where Jaccard distance is used to measure the set distance. As Fig.14 presents, there are five communities that do not share any common user and there are 9 communities when distance is set as 0.6. The results indicate that many MCCs have diverse set members. However, the problem in [3] only find one maximum MCC and ignore all others despite the fact that other MCCs are equally meaningful and very different from members in the maximum MCC.

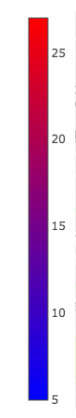

(a)

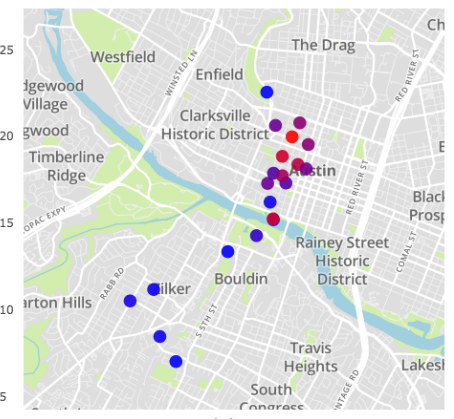

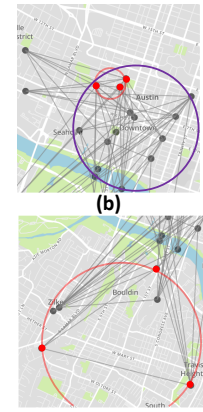

(c)
Figure 13: 2-MCCs detection and results of [11] .

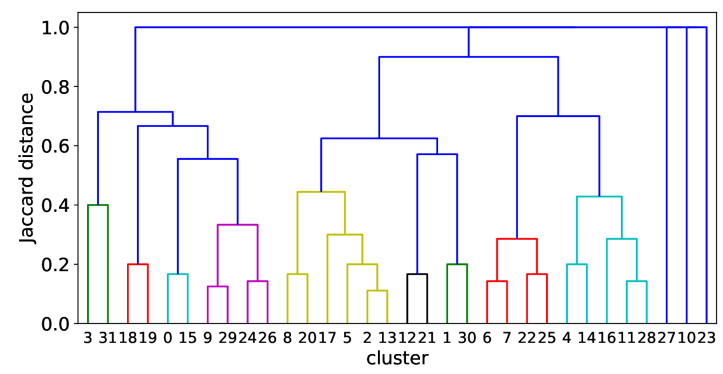

Figure 14: Clustering Dendrogram for MCCs.

\section{CONCLUSION}

In this paper, we investigate the $d$-MCCs detection problem on large scale geo-social networks. Unlike prior work that searches MCC for given query nodes or finds one maximal MCC, we solve a community detection problem which detects all communities satisfying both social and spatial cohesiveness constraints. To make our solution compatible with existing community detection techniques, we design a uniform framework so that existing techniques like $k$-core and $k$-truss decomposition can be easily plugged in. Besides generality and compatibility, our MCC detection framework improves efficiency thanks to our spatial constraint checking algorithms and several engineering level optimization. The effectiveness and efficiency of both the spatial algorithm and the whole MCC detection framework are demonstrated by using three real-world datasets and two synthetic datasets with various parameter settings.

\section{REFERENCES}

[1] Yong-Yeol Ahn, James P Bagrow, and Sune Lehmann. 2010. Link communities reveal multiscale complexity in networks. Nature 466, 7307 (2010), 761.

[2] Ulrik Brandes, Daniel Delling, Marco Gaertler, Robert Gorke, Martin Hoefer, Zoran Nikoloski, and Dorothea Wagner. 2007. On modularity clustering. IEEE Trans. on Knowledge and Data Engg. 20, 2 (2007), 172-188.

[3] Lu Chen, Chengfei Liu, Rui Zhou, Jianxin Li, Xiaochun Yang, and Bin Wang. 2018. Maximum co-located community search in large scale social networks. Proc. of the VLDB Endowment 11, 10 (2018), 1233-1246.

[4] Yu Chen, Jun $\mathrm{Xu}$, and Minzheng Xu. 2015. Finding community structure in spatially constrained complex networks. Int. F. of Geog. Info. Sc. 29, 6 (2015), 889-911.

[5] Eunjoon Cho, Seth A Myers, and Jure Leskovec. 2011. Friendship and mobility: user movement in location-based social networks. In Proc. of the 17th SIGKDD Conf. ACM, 1082-1090.

[6] Wanyun Cui, Yanghua Xiao, Haixun Wang, Yiqi Lu, and Wei Wang. 2013. Online search of overlapping communities. In Proc.of the Int. Conf. on SIGMOD. ACM, 277-288.

[7] Wanyun Cui, Yanghua Xiao, Haixun Wang, and Wei Wang. 2014. Local search of communities in large graphs. In Proc. of the Int. SIGMOD Conf. ACM, 991-1002.

[8] Purvi Jayesh Desai. 2016. PIRCNET: A Data Driven Approach to HIV Risk Analysis. Ph.D. Dissertation. Univ. of California San Diego.

[9] D Jack Elzinga and Donald W Hearn. 1972. The minimum covering sphere problem. Management science 19, 1 (1972), 96-104.

[10] Jack Elzinga and Donald W Hearn. 1972. Geometrical solutions for some minimax location problems. Transportation Science 6, 4 (1972), 379-394.

[11] Yixiang Fang, Reynold Cheng, Xiaodong Li, Siqiang Luo, and Jiafeng Hu. 2017. Effective community search over large spatial graphs. Proc. of the VLDB Endowment 10, 6 (2017), 709-720.

[12] Santo Fortunato. 2010. Community detection in graphs. Physics Reports 486, 3-5 (2010), 75-174.

[13] Xin Huang, Hong Cheng, Lu Qin, Wentao Tian, and Jeffrey Xu Yu. 2014. Querying k-truss community in large and dynamic graphs. In Proc. of the Int. Conf. on SIGMOD. ACM, 1311-1322.

[14] Xin Huang, Laks VS Lakshmanan, Jeffrey Xu Yu, and Hong Cheng. 2015. Approximate closest community search in networks. Proc. of the VLDB Endowment 9, 4 (2015), 276-287.

[15] Andrea Lancichinetti and Santo Fortunato. 2009. Community detection algorithms: a comparative analysis. Physical Review E 80, 5 (2009), 056117.

[16] Guoliang Li, Shuo Chen, Jianhua Feng, Kian-lee Tan, and Wen-syan Li. 2014. Efficient location-aware influence maximization. In Proceedings of the 2014 ACM SIGMOD international conference on Management of data. ACM, 87-98.

[17] Mark EJ Newman. 2004. Fast algorithm for detecting community structure in networks. Physical Review E 69, 6 (2004), 066133.

[18] Mauro Sozio and Aristides Gionis. 2010. The community-search problem and how to plan a successful cocktail party. In Proc. of the 16th Int. SIGKDD Conf. ACM, 939-948.

[19] Kai Wang, Xin Cao, Xuemin Lin, Wenjie Zhang, and Lu Qin. 2018. Efficient computing of radius-bounded k-cores. In 2018 IEEE 34th International Conference on Data Engineering (ICDE). IEEE, 233-244.

[20] Nadir Weibel, Purvi Desai, Lawrence Saul, Amarnath Gupta, and Susan Little. 2017. HIV risk on twitter: The ethical dimension of social media evidence-based prevention for vulnerable populations. In Proc. of the 50th Hawaii Int. Conf. on System Sciences.

[21] Scott White and Padhraic Smyth. 2005. A spectral clustering approach to finding communities in graphs. In Proc. of the Int. Conf. on Data Mining. SIAM, 274-285.

[22] Chen Zhang, Ying Zhang, Wenjie Zhang, Lu Qin, and Jianye Yang. 2019. Efficient Maximal Spatial Clique Enumeration. In 2019 IEEE 35th International Conference on Data Engineering (ICDE). IEEE, 878-889.

[23] Fan Zhang, Ying Zhang, Lu Qin, Wenjie Zhang, and Xuemin Lin. 2017. When engagement meets similarity: efficient $(\mathrm{k}, \mathrm{r})$-core computation on social networks. Proc. of the VLDB Endowment 10, 10 (2017), 998-1009. 